\title{
Multivariate analysis of risk factors for complications after loop ileostomy closure
}

\author{
Análisis multivariable de los factores de riesgo asociados a complicaciones posterior al \\ cierre de ileostomía en asa
}

\author{
Omar Vergara-Fernández, Mario Trejo-Avila* and Noel Salgado-Nesme \\ Departamento de Cirugía Colorectal, Instituto Nacional de Ciencias Médicas y Nutrición Salvador Zubirán, Ciudad de México, México
}

\begin{abstract}
Background: Despite the advantages of diverting loop ileostomy construction, it is related to complications. Objective: The aim of the study was to determine the risk factors for complications after loop ileostomy closure. Methods: Patients who underwent loop ileostomy closure from January 2010 to March 2018 were retrospectively analyzed. Multivariate logistic regression was used to determine the effect of the potential risk factors on the rate of each complication. Results: $A$ total of 136 patients underwent reversal. Indications for the initial operation were colorectal cancer (39.7\%), diverticulitis (25.7\%), idiopathic chronic ulcerative colitis (ICUC) (8.1\%), familial adenomatous polyposis (FAP) (7.4\%), and others (19.1\%). Multivariate analysis identified the following risk factors: type of incision (midline laparotomy) (odds ratio $[O R]=6.5$ ) for wound infection; treatment with immunomodulator $(O R=12.5)$ for anastomotic leak; history of FAP $(O R=9.8)$ for intestinal obstruction; previous use of immunomodulator $(O R=10.0)$ and performing reversal through midline incision $(O R=18.9)$ for reoperation; and $\geq 65$ years old $(O R=3.5)$ for medical complications. The rate of incisional hernia was $11 \%$, and the risk factors were time to closure < 3 months $(O R=6.4)$ and parastomal hernia $(O R=13.2)$. Conclusions: Several patient-related and surgical technique factors should be considered at the time of loop ileostomy closure to reduce post-operative morbidity.
\end{abstract}

KEY WORDS: Loop ileostomy. Ileostomy closure. Stoma reversal complications.

\section{Resumen}

Antecedentes: A pesar de las ventajas de la ileostomía en asa de derivación, múltiples complicaciones se han asociado a su uso. Objetivo: Determinar los factores de riesgo para presentar complicaciones tras el cierre de una ileostomía en asa. Método: Se realizó un análisis retrospectivo de los pacientes sometidos a cierre de ileostomía en asa de enero de 2010 a marzo de 2018 . Se determinaron los factores de riesgo utilizando regresión multivariable. Resultados: Se incluyeron 136 pacientes. Las indicaciones para cirugía fueron cáncer colorrectal (39.7\%), diverticulitis (25.7\%), colitis ulcerosa crónica idiopática (CUCI) (8.1\%), poliposis adenomatosa familiar (PAF) (7.4\%) y otras (19.1\%). Se identificaron los siguientes factores de riesgo: incisión en línea media (OR: 6.5) para infección de herida; tratamiento inmunomodulador (OR: 12.5) para fuga de anastomosis; antecedente de PAF (OR: 9.8) para oclusión intestinal; tratamiento inmunomodulador (OR: 10) e incisión en línea media (OR: 18.9) para reintervención; y edad $\geq$ 65 años (OR: 3.5) para complicaciones médicas. La frecuencia de hernia incisional fue del 11\%: < 3 meses para el cierre (OR: 6.4) y hernia parastomal (OR: 13.2). Conclusiones: Numerosos factores relacionados con el paciente y con la técnica quirúrgica deben de ser considerados al momento del cierre de la ileostomía en asa para reducir la morbilidad posoperatoria.

PALABRAS CLAVE: Cierre de ileostomía en asa. Complicaciones de ileostomía. lleostomía en asa.

\author{
Correspondence: \\ *Mario Trejo-Avila \\ Vasco de Quiroga, 15 \\ Col. Sección XVI, Del. Tlalpan \\ C.P. 14080 Ciudad de México, México \\ E-mail: mario.trejo.avila@gmail.com
}

Date of reception: 20-07-2018

Date of acception: 24-08-2018

DOI: 10.24875/CIRU.18000611
Cir Cir. 2019;87:337-346

Contents available at PubMed www.cirugiaycirujanos.com 


\section{Introduction}

Diverting loop ileostomy is commonly performed to protect a distal anastomosis when there is a high risk of anastomotic leakage $\mathrm{e}^{1,2}$.

Although fecal diversion does not prevent an anastomotic leak, it does decrease the potential morbidity and mortality from an anastomotic leak ${ }^{3}$. Despite the potential benefits of fecal diversion, loop ileostomy construction is related to a significant rate of complications.

Closure of the loop ileostomy is associated with a low mortality, but morbidity may be as high as $20 \% 4$. Patients needed additional operation (closure surgery) and may develop small bowel obstructions, parastomal hernia, stoma retraction or prolapse, skin problems, electrolyte abnormalities, and acute kidney injury due to a high output stoma ${ }^{3,5}$.

Furthermore, stoma reversal may cause several complications, such as surgical site infection (SSI), anastomotic leak, bowel obstruction, post-operative ileus, wound dehiscence, and enterocutaneous fistulas $\mathrm{s}^{6,7}$. There is also an increased risk of developing an incisional hernia after stoma closure ${ }^{5}$.

The aim of the study was to determine the risk factors for complications after loop ileostomy closure using multivariate analysis, either if the first procedure was for a benign or a malignant colorectal disease.

\section{Patients and methods}

From January 2010 to March 2018, all patients who underwent loop ileostomy closure at Instituto Nacional de Ciencias Médicas y Nutrición Salvador Zubirán in Mexico City, Mexico, were included in this study. Data for these patients were retrospectively retrieved and analyzed.

The inclusion criteria included patients who were $\geq 18$ years old and who underwent planned loop ileostomy closure. Patients included had previous benign or malignant colorectal disease. Index surgery was defined as the operation that included colorectal resection (for benign or malignant disease), primary anastomosis, and construction of diverting loop ileostomy. The indications for protective loop ileostomy construction were low colorectal, ileorectal, coloanal, or ileal pouch anastomoses.

Exclusion criteria were patients younger than 18, patients who had diverting loop ileostomy non-related to colorectal diseases or operations, and patients with double-barrel or terminal ileostomy. Patients with incomplete data were eliminated from the study.

All patients included in the study had pre-operative water-soluble contrast enema and colonoscopic evaluation before the loop ileostomy closure. All anastomoses were side-to-side and were performed with linear staplers and reinforced with non-absorbable sutures.

Ileostomy closure was performed either by peristomal incision (circular incision around the ileostomy) or by a midline incision as required. All patients had a peristomal incision first approach, and some patients needed conversion to midline incision mainly due to dense adhesions or inadequate small bowel length obtained through peristomal incision. Closure of the ostomy wound was performed by conventional linear closure or by pursestring closure, based on surgeons' preference.

Pre-operative variables considered in this study were sex (female and male), body mass index $(<30$ or $\geq 30$ ), age $(<65$ or $\geq 65$ ), and the American Society of Anesthesiology grade (ASA I-II or III-IV). Comorbidity was assessed using the Charlson Comorbidity Index $(\mathrm{CCl})^{8}$. Other pre-operative variables were tobacco use and previous treatments (systemic steroids, immunomodulators, radiotherapy, and chemotherapy). Immunomodulators included azathioprine, methotrexate, and mycophenolate mofetil. Either immunomodulators or systemic steroids were withdrawn before the loop ileostomy closure. Variables regarding index surgery were type of index surgery (elective or urgent and laparoscopic or open) and reintervention for complications after index surgery. Surgical variables concerning loop ileostomy closure were interval from index surgery to closure (months), type of incision (peristomal or midline), and peristomal wound closure (linear or purse-string).

The following complications after loop ileostomy closure were analyzed: wound infection either deep or superficial, anastomotic leakage, intestinal obstruction, reintervention after loop ileostomy closure, overall medical complications, and mortality. All post-operative complications were recorded and stratified in accordance with Clavien-Dindo classification scheme (occurring either within 30 days of loop ileostomy closure or during the hospital stay) $)^{9}$. The rate of incisional hernia was also analyzed in the study.

Wound infections were either superficial or deep infections, based on the definition of the Centers for Disease Control for Surgical Site Infections ${ }^{10}$. Anastomotic leakage was confirmed with clinical and 
tomographic evidence of leak or perianastomotic collection. Intestinal obstruction was diagnosed in patients with clinical symptoms and signs of obstruction (distention, pain, vomiting, and absence of gas/stool passage) and corroborated by imaging (dilated small bowel). Reintervention was the need to return to the operation room secondary to a complication directly related to the loop ileostomy closure and included all complications occurring within 30 days after loop ileostomy closure.

Incisional hernia repair was not considered in the reintervention group and was not included in the Clavien-Dindo classification. Incisional hernia was diagnosed by clinical examination and corroborated by computed tomography. For incisional hernia, a period of 1 year to follow-up after ileostomy closure was considered in the analysis.

\section{Statistical Analysis}

All data were collected retrospectively in a digital database. Categorical data were presented as totals (n) and proportions as percentages (\%). Categorical data were compared using the Pearson's Chi-square test or Fisher's exact test. All tests were two-sided and used an alpha of 0.05 .

Univariate analysis was performed for each complication (analyzing patients in two groups: complication present vs. absence of complication). All variables with a $p$ value inferior to 0.10 in the univariate analysis were considered as potential risk factors and were entered into multivariate backward logistic regression analysis. The variables representing the lowest risk for each complication was considered to be the reference group (odds ratio $[O R]=1$ ). OR and $95 \%$ confidence intervals $(95 \% \mathrm{Cl})$ were calculated for each outcome (complication) (wound infection, anastomotic leakage, intestinal obstruction, incisional hernia, reintervention after ileostomy closure, overall medical complications, and mortality). $p<0.05$ was considered to be statistically significant. All data were analyzed using SPSS statistic version 22.0 (IBM Corporation, Armonk, New York, NY).

\section{Results}

From January 2010 to March 2018, a total of 136 patients underwent loop ileostomy closure at our institution. Patient demographic and clinical characteristics are summarized in table 1 . There were 69 women $(50.7 \%), 71.3 \%(n=97)$ of patients were $<65$ years old, $91.9 \%(n=125)$ had a BMl $<30 \mathrm{~kg} / \mathrm{m}^{2}$, and $58.1 \%$ ( $n=79)$ had a $\mathrm{CCl} \geq 2$.

Of the 136 patients, $82(60.3 \%)$ had benign disease and $54(39.7 \%)$ had malignant colorectal disease.

Patients in the benign disease group had one of the following diagnosis: complicated diverticular disease $(n=35)$, ulcerative colitis $(n=11)$, familial adenomatous polyposis (FAP) syndrome $(n=10)$, rectovesical fistula $(n=8)$, rectovaginal fistula $(n=8)$, colorectal trauma $(n=5)$, Hirschsprung's disease $(n=1)$, ischemic colitis $(n=2)$, colonic angiodysplasia $(n=2)$, rectal prolapse $(n=1)$, and obstructing rectal adenoma $(n=2)$.

Patients with malignant disease had rectal cancer $(n=40)$ or colon cancer $(n=14)$. The distribution of patients according to cancer stage (TNM) was ${ }^{11}$ Stage I ( $n=7,12.9 \%)$, II $(n=8,14.8 \%)$, III $(n=28$, $51.8 \%)$, and IV ( $n=11,20.3 \%)$.

Comparisons of baseline pre-operative data, previous treatments, characteristics of the index surgery, and perioperative data concerning loop ileostomy closure are shown in table 1.

\section{Wound infection}

The overall rate of wound infection (superficial and deep) was $6.6 \%$. No statistically significant difference exists between patients with benign and malignant disease for the rate of wound infection $(8.5 \%$ vs. $3.7 \%$, $p=0.267)$ (Table 2).

Univariate analysis results are summarized in table 3. Previous radiotherapy, chemotherapy, midline incision, and linear wound closure were considered potential risk factors for the occurrence of wound infection $(p<0.1)$.

Multivariate analysis identified the type of incision (conversion to midline laparotomy) as the only risk factor $(\mathrm{OR}=6.5[95 \% \mathrm{Cl} 1.29-32.70] ; p=0.023)$.

\section{Anastomotic leakage}

The overall rate of anastomotic leakage was $5.1 \%$. Anastomotic leakage was similar between patients with baseline benign and malignant disease $(2.4 \%$ vs. 9.3\%; $p=0.078$ ) (Table 2).

Univariate analysis showed that previous treatment with immunomodulator, malignant disease, and midline incision could be considered as potential risk factors $(p<0.1)$ for anastomotic leakage (Table 3). Multivariate analysis identified previous treatment with 
Table 1. Characteristics of patients

\begin{tabular}{|c|c|c|c|c|}
\hline & $\begin{array}{l}\text { All patients } \\
(n=136)\end{array}$ & $\begin{array}{l}\text { Benign disease } \\
\quad(n=82)\end{array}$ & $\begin{array}{l}\text { Malignant disease } \\
\qquad(n=54)\end{array}$ & $p<0.05$ \\
\hline $\begin{array}{l}\text { Sex } \\
\text { Female } \\
\text { Male }\end{array}$ & $\begin{array}{l}69(50.7) \\
67(49.3)\end{array}$ & $\begin{array}{l}41(50) \\
41(50)\end{array}$ & $\begin{array}{l}28(51.9) \\
26(48.1)\end{array}$ & 0.833 \\
\hline $\begin{array}{l}\text { Age, } n(\%) \\
<65 \text { years } \\
\geq 65 \text { years }\end{array}$ & $\begin{array}{l}97(71.3) \\
39(28.7)\end{array}$ & $\begin{array}{l}62(75.6) \\
20(24.4)\end{array}$ & $\begin{array}{l}35(64.8) \\
19(35.2)\end{array}$ & 0.173 \\
\hline $\begin{array}{l}\text { BMI, n }(\%) \\
\quad<30 \\
\geq 30\end{array}$ & $\begin{array}{c}125(91.9) \\
11(8.1)\end{array}$ & $\begin{array}{c}74(90.2) \\
8(9.8)\end{array}$ & $\begin{array}{c}51(94.4) \\
3(5.6)\end{array}$ & 0.379 \\
\hline $\begin{array}{l}\text { Smoking, n (\%) } \\
\text { Yes } \\
\text { No }\end{array}$ & $\begin{array}{l}56(41.2) \\
80(58.8)\end{array}$ & $\begin{array}{l}34(41.5) \\
48(58.5)\end{array}$ & $\begin{array}{l}22(40.7) \\
32(59.3)\end{array}$ & 0.933 \\
\hline $\begin{array}{l}\text { Charlson Comorbidity Index } \\
0-1 \\
2-3 \\
4-5 \\
\geq 6\end{array}$ & $\begin{array}{c}57(41.9) \\
41(30.1) \\
31(22.8) \\
7(5.1)\end{array}$ & $\begin{array}{l}48(58.5) \\
19(23.2) \\
15(18.3) \\
0\end{array}$ & $\begin{array}{c}9(16.7) \\
22(40.7) \\
16(29.6) \\
7(13)\end{array}$ & $<0.001$ \\
\hline $\begin{array}{l}\text { ASA classification } \\
\text { I-II } \\
\text { III-IV }\end{array}$ & $\begin{array}{l}102(75) \\
34(25)\end{array}$ & $\begin{array}{l}66(80.5) \\
16(19.5)\end{array}$ & $\begin{array}{l}36(66.7) \\
18(33.3)\end{array}$ & 0.069 \\
\hline $\begin{array}{l}\text { Previous treatments, n (\%) } \\
\text { Steroids } \\
\text { Immunomodulator } \\
\text { Radiotherapy } \\
\text { Chemotherapy }\end{array}$ & $\begin{array}{c}21(15.4) \\
6(4.4) \\
35(25.7) \\
43(31.6)\end{array}$ & $\begin{array}{c}19(23.2) \\
6(7.3) \\
0 \\
1(1.2)\end{array}$ & $\begin{array}{c}2(3.7) \\
0 \\
35(64.8) \\
42(77.8)\end{array}$ & $\begin{array}{l}0.002 \\
0.042 \\
<0.001 \\
<0.001\end{array}$ \\
\hline $\begin{array}{l}\text { Index surgery, n (\%) } \\
\text { Elective } \\
\text { Urgent }\end{array}$ & $\begin{array}{l}91(66.9) \\
45(33.1)\end{array}$ & $\begin{array}{l}41(50) \\
41(50)\end{array}$ & $\begin{array}{c}50(92.6) \\
4(7.4)\end{array}$ & $<0.001$ \\
\hline $\begin{array}{l}\text { Modality of index surgery, n (\%) } \\
\text { Laparoscopic } \\
\text { Open }\end{array}$ & $\begin{array}{l}36(26.5) \\
100(73.5)\end{array}$ & $\begin{array}{l}10(12.2) \\
72(87.8)\end{array}$ & $\begin{array}{l}26(48.1) \\
28(51.9)\end{array}$ & $<0.001$ \\
\hline $\begin{array}{l}\text { Reintervention after index } \\
\text { surgery, } \mathrm{n}(\%) \\
\text { Yes } \\
\text { No }\end{array}$ & $\begin{array}{c}30(22.1) \\
106(77.9)\end{array}$ & $\begin{array}{l}19(23.3) \\
63(76.8)\end{array}$ & $\begin{array}{l}11(20.4) \\
43(79.6)\end{array}$ & 0.700 \\
\hline $\begin{array}{l}\text { Interval from index surgery } \\
\text { to loop closure, } \mathrm{n}(\%) \\
\leq 3 \text { months } \\
3-6 \text { months } \\
\geq 6 \text { months }\end{array}$ & $\begin{array}{l}27(19.9) \\
48(35.3) \\
61(44.9)\end{array}$ & $\begin{array}{l}19(23.2) \\
33(40.2) \\
30(36.6)\end{array}$ & $\begin{array}{l}8(14.8) \\
15(27.8) \\
31(57.4)\end{array}$ & 0.057 \\
\hline $\begin{array}{l}\text { Parastomal hernia, n (\%) } \\
\text { Yes } \\
\text { No }\end{array}$ & $\begin{array}{c}13(9.6) \\
123(90.4)\end{array}$ & $\begin{array}{l}10(12.2) \\
72(87.8)\end{array}$ & $\begin{array}{c}3(5.6) \\
51(94.4)\end{array}$ & 0.322 \\
\hline $\begin{array}{l}\text { Type of incision, n (\%) } \\
\text { Peristomal } \\
\text { Midline }\end{array}$ & $\begin{array}{c}123(90.4) \\
13(9.6)\end{array}$ & $\begin{array}{l}75(91.5) \\
7(8.5)\end{array}$ & $\begin{array}{l}48(88.9) \\
6(11.1)\end{array}$ & 0.617 \\
\hline $\begin{array}{l}\text { Wound closure, n (\%) } \\
\text { Linear } \\
\text { Purse string }\end{array}$ & $\begin{array}{l}79(58.1) \\
57(41.9)\end{array}$ & $\begin{array}{l}47(57.3) \\
35(42.7)\end{array}$ & $\begin{array}{l}32(59.3) \\
22(40.7)\end{array}$ & 0.822 \\
\hline
\end{tabular}

BMI: body mass index (kg/m²); ASA: American Society of Anesthesiology. 
Table 2. Complications after loop ileostomy closure

\begin{tabular}{|c|c|c|c|c|}
\hline & $\begin{array}{l}\text { All patient } \\
(n=136)\end{array}$ & $\begin{array}{l}\text { Benign disease } \\
\quad(n=82)\end{array}$ & $\begin{array}{l}\text { Malignant disease } \\
\quad(n=54)\end{array}$ & $\mathrm{p}<0.05$ \\
\hline \multicolumn{5}{|l|}{$\begin{array}{l}\text { 30-day post-operative complications } \\
\text { (Clavien-Dindo) } \\
\text { । }\end{array}$} \\
\hline Urinary retention & $2(1.5)$ & $2(2.4)$ & 0 & 0.517 \\
\hline Fever & $2(1.5)$ & $1(1.2)$ & $1(1.9)$ & 0.764 \\
\hline \multicolumn{5}{|l|}{ II } \\
\hline Urinary tract infection & $2(1.5)$ & $1(1.2)$ & $1(1.9)$ & 0.764 \\
\hline Delirium & $2(1.5)$ & $2(2.4)$ & 0 & 0.517 \\
\hline Pneumonia & $2(1.5)$ & $1(1.2)$ & $1(1.9)$ & 0.764 \\
\hline CD diarrhea & $1(0.7)$ & 0 & $1(1.9)$ & 0.397 \\
\hline Wound infection & $9(6.6)$ & $7(8.5)$ & $2(3.7)$ & 0.267 \\
\hline Intestinal obstruction & $11(8)$ & $8(9.7)$ & $3(5.5)$ & 0.379 \\
\hline \multicolumn{5}{|l|}{ Illa } \\
\hline Abdominal fluid collection & $2(1.5)$ & $1(1.2)$ & $1(1.9)$ & 0.764 \\
\hline \multicolumn{5}{|l|}{ Illb } \\
\hline Intra-abdominal abscess & $1(0.7)$ & $1(1.2)$ & 0 & 1.000 \\
\hline Intestinal obstruction & $2(1.5)$ & $1(1.2)$ & $1(1.9)$ & 0.764 \\
\hline Anastomotic leakage & $7(5.1)$ & $2(2.4)$ & $5(9.3)$ & 0.078 \\
\hline \multicolumn{5}{|l|}{ IV } \\
\hline Myocardial infarction (mortality) & $2(1.5)$ & $1(1.2)$ & $1(1.9)$ & 0.764 \\
\hline Incisional hernia, n (\%) & & & & 0.098 \\
\hline Yes & $15(11)$ & $12(14.6)$ & $3(5.6)$ & \\
\hline No & $121(89)$ & $70(85.4)$ & $51(37.5)$ & \\
\hline
\end{tabular}

ACS: acute coronary syndrome, SSI: surgical site infections. CD diarrhea: clostridium difficile diarrhea.

Table 3. Univariate and multivariate analysis of factors associated with post-operative complications

\begin{tabular}{|c|c|c|c|c|c|}
\hline \multirow[b]{2}{*}{ Factors } & \multicolumn{3}{|c|}{ Univariate analysis } & \multicolumn{2}{|c|}{$\begin{array}{l}\text { Multivariate logistic } \\
\text { regression }\end{array}$} \\
\hline & $\begin{array}{l}\text { Wound infection } \\
\qquad(\mathrm{n}=9)\end{array}$ & $\begin{array}{l}\text { No wound infection } \\
\qquad(n=127)\end{array}$ & $p$ value & OR (95\% Cl) & $\begin{array}{c}p \\
\text { value }\end{array}$ \\
\hline $\begin{array}{l}\text { Previous radiotherapy } \\
\text { Yes } \\
\text { No }\end{array}$ & $\begin{array}{c}0 \\
9(100)\end{array}$ & $\begin{array}{l}35(27.6) \\
92(72.4)\end{array}$ & 0.068 & & \\
\hline $\begin{array}{l}\text { Previous chemotherapy } \\
\text { Yes } \\
\text { No }\end{array}$ & $\begin{array}{c}0 \\
9(100)\end{array}$ & $\begin{array}{l}43(33.9) \\
84(66.1)\end{array}$ & 0.035 & & \\
\hline $\begin{array}{l}\text { Type of incision } \\
\text { Peristomal } \\
\text { Midline }\end{array}$ & $\begin{array}{l}6(66.7) \\
3(33.3)\end{array}$ & $\begin{array}{c}117(92.1) \\
10(7.9)\end{array}$ & 0.012 & $\begin{array}{c}1.00 \\
6.5(1.29-32.70)\end{array}$ & 0.023 \\
\hline $\begin{array}{l}\text { Wound closure } \\
\text { Purse string } \\
\text { Linear }\end{array}$ & $\begin{array}{l}1(11.1) \\
8(88.9)\end{array}$ & $\begin{array}{l}56(44.1) \\
71(55.9)\end{array}$ & 0.053 & & \\
\hline Factors & $\begin{array}{l}\text { Anastomotic leak } \\
\qquad(\mathrm{n}=7)\end{array}$ & $\begin{array}{l}\text { No anastomotic leak } \\
\qquad(n=129)\end{array}$ & $p$ value & OR (95\% Cl) & $\begin{array}{c}p \\
\text { value }\end{array}$ \\
\hline $\begin{array}{l}\text { Immunomodulator } \\
\text { Yes } \\
\text { No }\end{array}$ & $\begin{array}{l}2(28.6) \\
5(71.4)\end{array}$ & $\begin{array}{c}4(3.1) \\
125(96.9)\end{array}$ & 0.001 & $\begin{array}{c}12.5(1.83-85.12) \\
1.00\end{array}$ & 0.010 \\
\hline $\begin{array}{l}\text { Primary disease } \\
\text { Benign } \\
\text { Malignant }\end{array}$ & $\begin{array}{l}2(28.6) \\
5(71.4)\end{array}$ & $\begin{array}{l}80(62) \\
49(38)\end{array}$ & 0.078 & & \\
\hline $\begin{array}{l}\text { Type of incision } \\
\text { Peristomal } \\
\text { Midline }\end{array}$ & $\begin{array}{l}5(71.4) \\
2(28.6)\end{array}$ & $\begin{array}{c}118(91.5) \\
11(8.5)\end{array}$ & 0.079 & & \\
\hline
\end{tabular}


Table 3. Univariate and multivariate analysis of factors associated with post-operative complications (Continued)

\begin{tabular}{|c|c|c|c|c|c|}
\hline Factors & $\begin{array}{l}\text { Intestinal obstruction } \\
\qquad(n=13)\end{array}$ & $\begin{array}{l}\text { No intestinal obstruction } \\
\qquad(n=123)\end{array}$ & $p$ value & OR $(95 \% \mathrm{Cl})$ & $\begin{array}{c}\mathrm{p} \\
\text { value }\end{array}$ \\
\hline $\begin{array}{l}\text { FAP } \\
\text { Yes } \\
\text { No }\end{array}$ & $\begin{array}{l}4(30.8) \\
9(69.2)\end{array}$ & $\begin{array}{c}6(4.9) \\
117(95.1)\end{array}$ & 0.001 & $\begin{array}{c}9.8(2.10-46.41) \\
1.00\end{array}$ & 0.004 \\
\hline $\begin{array}{l}\text { Reintervention after index surgery } \\
\text { Yes } \\
\text { No }\end{array}$ & $\begin{array}{c}0 \\
13(100)\end{array}$ & $\begin{array}{l}30(24.4) \\
93(75.6)\end{array}$ & 0.044 & & \\
\hline Factors & $\begin{array}{l}\text { Incisional hernia } \\
\qquad(n=15)\end{array}$ & $\begin{array}{l}\text { No incisional hernia } \\
\quad(n=121)\end{array}$ & $p$ value & OR $(95 \% \mathrm{Cl})$ & $\begin{array}{c}\mathrm{p} \\
\text { value }\end{array}$ \\
\hline $\begin{array}{l}\text { BMl } \\
\quad<30 \\
\geq 30\end{array}$ & $\begin{array}{l}12(80) \\
3(20)\end{array}$ & $\begin{array}{c}113(93.4) \\
8(6.6)\end{array}$ & 0.073 & & \\
\hline $\begin{array}{l}\text { Primary disease } \\
\text { Benign } \\
\text { Malignant }\end{array}$ & $\begin{array}{l}12(80) \\
3(20)\end{array}$ & $\begin{array}{l}70(57.9) \\
51(42.1)\end{array}$ & 0.098 & & \\
\hline $\begin{array}{l}\text { Type of index surgery } \\
\text { Elective } \\
\text { Urgent }\end{array}$ & $\begin{array}{l}7(46.7) \\
8(53.3)\end{array}$ & $\begin{array}{l}84(69.4) \\
37(30.6)\end{array}$ & 0.077 & & \\
\hline $\begin{array}{l}\text { Time to loop closure } \\
<3 \text { months } \\
>3 \text { months }\end{array}$ & $\begin{array}{l}6(40) \\
9(60)\end{array}$ & $\begin{array}{l}21(17.4) \\
100(82.6)\end{array}$ & 0.038 & $\begin{array}{c}6.4(1.58-26.14) \\
1.00\end{array}$ & 0.009 \\
\hline $\begin{array}{l}\text { Parastomal hernia } \\
\text { Yes } \\
\text { No }\end{array}$ & $\begin{array}{c}5(33.3) \\
10(66.7)\end{array}$ & $\begin{array}{c}8(6.6) \\
113(93.4)\end{array}$ & 0.001 & $\begin{array}{c}13.2(2.70-64.58) \\
1.00\end{array}$ & 0.001 \\
\hline
\end{tabular}

For multivariable logistic regression analysis, OR and $95 \% \mathrm{Cl}$ are presented. OR are calculated for each outcome (SSI), anastomotic leak, intestinal obstruction, and incisional hernia. Only significant results are shown. The reference category has an OR of 1.00 .

OR: odds ratio, Cl: confidence interval, SSI: surgical site infection.

immunomodulator as the only risk factor for developing anastomotic leak $(\mathrm{OR}=12.5(95 \% \mathrm{Cl}=1.83-85.12)$; $p=0.010)$.

\section{Intestinal obstruction}

Intestinal obstruction was developed in $9.5 \%$ of the patients following loop ileostomy closure (Table 2). Of the total of patients with this diagnosis, $11(8 \%)$ resolved with nasogastric tube and nil per os, and 2 (1.5\%) patients needed reoperation.

Univariate analysis revealed that reintervention after index surgery as well as a history of FAP syndrome was associated with the occurrence of intestinal obstruction after loop ileostomy closure (Table 3).

Multivariate logistic regression analysis identified a history of FAP syndrome as the only risk factor $(\mathrm{OR}=9.8[95 \% \mathrm{Cl} 2.10-46.41] ; \mathrm{p}=0.004)$.

\section{Reoperation}

The overall reoperation rate after loop ileostomy closure was $7.3 \%$. Reoperations were needed due to intestinal obstruction (1.5\%), anastomotic leak (5.1\%), and intra-abdominal abscess $(0.7 \%)$ (Table 2$)$.

Univariate analysis showed that females, previous use of immunomodulator, and performing ileostomy closure through midline incision were associated with the need of reoperation.

Multivariate analysis determined two independent risk factors for reoperation: previous use of immunomodulator $(\mathrm{OR}=10.0[95 \% \mathrm{Cl} 1.33-75.66] ; \mathrm{p}=0.025)$ and performing ileostomy closure through midline incision $(\mathrm{OR}=18.9[95 \% \mathrm{Cl} 3.37-106.62] ; p=0.001)$ (Table 4).

\section{Incisional hernia}

The rate of incisional hernia was $11 \%$, being more frequent in patients with benign colorectal disease, although this difference was not statistically significant (14.6\% vs. $5.6 \%, p=0.098$ ) (Table 2).

Univariate analysis revealed that body mass index higher than 30 , history of benign colorectal disease, index surgery performed in urgent basis, time to loop ileostomy closure within 3 months from index surgery, 
Table 4. Univariate and multivariate analysis of factors associated with post-operative complications

\begin{tabular}{|c|c|c|c|c|c|}
\hline \multirow[b]{2}{*}{ Factors } & \multicolumn{3}{|c|}{ Univariate analysis } & \multicolumn{2}{|c|}{ Multivariate logistic regression } \\
\hline & $\begin{array}{l}\text { Reoperation } \\
\quad(n=10)\end{array}$ & $\begin{array}{l}\text { No reoperation } \\
\quad(n=126)\end{array}$ & $p$ value & OR $(95 \% \mathrm{Cl})$ & $p$ value \\
\hline Sex & & & 0.054 & & \\
\hline Female & $8(80)$ & $61(48.4)$ & & & \\
\hline Male & $2(20)$ & $65(51.6)$ & & & \\
\hline Immunomodulator & & & 0.013 & & 0.025 \\
\hline Yes & $2(20)$ & $4(3.2)$ & & $10.0(1.33-75.66)$ & \\
\hline No & $8(80)$ & $122(96.8)$ & & 1.00 & \\
\hline Type of incision & & & 0.001 & & 0.001 \\
\hline Peristomal & $6(60)$ & 117 (92.9) & & 1.00 & \\
\hline Midline & $4(40)$ & $9(7.1)$ & & $18.9(3.37-106.62)$ & \\
\hline Factors & $\begin{array}{l}\text { Medical complications } \\
\qquad(n=13)\end{array}$ & $\begin{array}{l}\text { No medical complications } \\
\qquad(n=123)\end{array}$ & $p$ value & OR $(95 \% \mathrm{Cl})$ & $p$ value \\
\hline Age & & & 0.035 & & 0.041 \\
\hline$<65$ & $6(46.2)$ & $91(74)$ & & 1.00 & \\
\hline$\geq 65$ & $7(53.8)$ & $32(26)$ & & $3.5(1.05-11.70)$ & \\
\hline Type of incision & & & 0.081 & & \\
\hline Peristomal & $10(76.9)$ & $113(91.9)$ & & & \\
\hline Midline & $3(23.1)$ & $10(8.1)$ & & & \\
\hline Wound closure & & & 0.009 & & 0.027 \\
\hline Linear & $12(92.3)$ & $67(54.5)$ & & $10.4(1.30-84.22)$ & \\
\hline Pursestring & $1(7.7)$ & $56(45.5)$ & & 1.00 & \\
\hline Factors & $\begin{array}{l}\text { Mortality } \\
(\mathrm{n}=2)\end{array}$ & $\begin{array}{l}\text { No mortality } \\
(n=126)\end{array}$ & $p$ value & OR $(95 \% \mathrm{Cl})$ & $p$ value \\
\hline Age & & & 0.025 & & \\
\hline$<65$ & 0 & $97(72.4)$ & & & \\
\hline$\geq 65$ & $2(100)$ & $37(27.6)$ & & & \\
\hline ASA classification & & & 0.014 & & \\
\hline I-II & 0 & $102(76.1)$ & & & \\
\hline III-IV & $2(100)$ & $32(23.9)$ & & & \\
\hline $\mathrm{CCl}$ & & & 0.018 & & \\
\hline $0-1$ & 0 & $57(42.5)$ & & & \\
\hline $2-3$ & 0 & $41(30.6)$ & & & \\
\hline $4-5$ & $1(50)$ & $30(22.4)$ & & & \\
\hline$\geq 6$ & $1(50)$ & $6(4.5)$ & & & \\
\hline
\end{tabular}

$\mathrm{CCl}$. For multivariable logistic regression analysis, OR and $95 \% \mathrm{Cl}$ are presented. ORs were calculated for each outcome (reoperation and medical complications). Only significant results are shown. The reference category has an OR of 1.00. OR: odds ratio, Cl: confidence interval, CCl: Charlson Comorbidity Index.

and presence of parastomal hernia were considered as potential risk factors for incisional hernia (Table 3).

Multivariate analysis identified two independent risk factors for incisional hernia: time to loop ileostomy closure within 3 months from index surgery $(\mathrm{OR}=6.4$ [95\% Cl 1.58-26.14]; $p=0.009$ ) and presence of parastomal hernia $(\mathrm{OR}=13.2[95 \% \mathrm{Cl} 2.70-64.58] ; p=0.001)$.

\section{Medical complications}

The overall rate of medical complications was $9.5 \%$. Several complications were considered as being medical and are shown in Table 2.
Univariate analysis identified age $\geq 65$ years old, midline incision, and peristomal linear wound closure as potential risk factors (Table 4).

Multivariate analysis determined two independent risk factors for medical complications: $\geq 65$ years old $(\mathrm{OR}=3.5$ [95\% Cl 1.05-11.70]; $p=0.041$ ) and linear wound closure $(\mathrm{OR}=10.4$ [95\% Cl 1.30-84.22]; $p=0.027)$.

\section{Mortality}

The mortality rate found in our study was $1.5 \%$. Based on univariate analysis, age > 65 years $(p=0.025)$, ASA grade III-IV ( $p=0.014)$, and Charlson 
morbidity index $>4(p=0.018)$ were found to be associated with mortality. None of these factors resulted significantly on multivariate regression analysis (Table 4).

\section{Discussion}

There is a trend toward sphincter-sparing procedures with the use of very low pelvic anastomoses in either rectal cancer or benign conditions (diverticulitis, FAP, or ulcerative colitis) ${ }^{12}$.

The most dreaded complication of a low (pelvic) anastomosis is an anastomotic leak. Anastomotic leakage could result in generalized peritonitis or pelvic abscess, long length of hospital stay, decreased quality of life, cancer recurrence, and higher mortality ${ }^{1,13,14}$. Fecal diversion is aimed to minimize these complications $\mathrm{s}^{1,13}$.

The incidence of anastomotic leakage is higher if it is distal in the rectum or if there are predisposing factors such as immunosuppression, history of chemo- and radiotherapy, acute sepsis, and poor nutritional status ${ }^{1,3}$. Other factors related to leaks are smoking, obesity, transfusion, and ASA class III-IV3,15. The incidence of anastomotic leak after colorectal surgery ranges from $1 \%$ to $25 \%{ }^{12,16}$ and the mortality ranges from $6 \%$ to $22 \%{ }^{17}$.

The absence of a diverting stoma in low anterior resection is associated with significant higher rate or anastomotic leak and reintervention ${ }^{18}$. For ultralow resections with subsequent coloanal anastomosis, diverting ostomy is almost always constructed ${ }^{3}$.

Despite the advantages of loop ileostomy, the role of temporary fecal diversion remains an area of controversy among surgeons ${ }^{3}$. Controversy rises because the significant morbidity and morality related to ileostomy (high output ileostomy, acute renal failure, parastomal herniation) and to ileostomy reversal (surgical site infections, anastomotic leak). Furthermore, it has been estimated that $40-50 \%$ of temporary stomas are never reversed ${ }^{12}$.

Morbidity and mortality related to loop ileostomy closure had been described in several series as being $3-30 \%^{1,19}$ and $0-4 \%^{1,20}$, respectively.

In a large cohort of patients (from the NSQIP database) who underwent closure of ileostomy, $9.3 \%$ of patients had major complications, $8.4 \%$ had minor complications, and there was a mortality rate of $0.6 \%{ }^{1}$. Functional status of the patient, ASA class, and organ dysfunction were independent predictors of complications after ileostomy reversal'.
In other study, elderly patient with age $>80$ years $(\mathrm{OR}=4.3[1.6-11.0] ; p=0.003)$ was the only independent risk factor for post-operative complications on multivariate analysis ${ }^{13}$.

Mennigen et al. ${ }^{7}$, in a systematic review of risks of ileostomy closure after restorative proctocolectomy for ulcerative colitis and FAP $(n=2146)$, reported that an overall morbidity was $16.5 \%$, reoperation rate was $3 \%$, anastomotic leak was $2.0 \%$, bowel obstruction was $7.6 \%$ (with $2.9 \%$ requiring laparotomy for this), wound infection rate was $4.0 \%$, and stoma site hernias was $1.9 \%$.

On regard of the anastomotic leak, in a recent meta-analysis, there was no difference in the rate of anastomotic leak between the hand-sewn and the stapled anastomotic techniques for closure of a loop ileostomy (OR 0.81, 95\% Cl 0.43-1.54, p = 0.52, $\left.1^{2}=33 \%\right)^{21}$. Saha et al. ${ }^{22}$ reported a $4 \%$ rate of anastomotic leak, with pre-operative anemia being significantly associated with leakage $(p=0.033)$.

Man et al..$^{13}$ determined that closure of the ileostomy with hand-sewn techniques showed a higher incidence of post-operative intestinal obstruction ( $p=0.049)$ compared to closure using stapler. This finding was corroborated in a meta-analysis, being higher the rate of small-bowel obstruction in the hand-sewn group compared to the stapled group $(7.03 \%$ vs. $5.58 \%$; OR $\left.0.69,95 \% \mathrm{Cl} 0.51-0.92, \mathrm{p}=0.01, \mathrm{l}^{2}=0 \%\right)^{21}$.

Surgical site infection following stoma reversal increases the risk of wound dehiscence, incisional hernia, length of stay, and health-care costs ${ }^{6}$. In a recent meta-analysis (including ileostomy and colostomy reversal), pursestring closure had a significant decrease in SSI (risk difference, $-0.25 ; 95 \% \mathrm{Cl},-0.36-0.15$; $p<0.00001$; number needed to treat $=4$ ) and higher satisfaction with cosmetic outcomes (standard mean difference, $0.7 ; 95 \% \mathrm{Cl}, 0.13-1.27 ; p=0.02$ ), when compared with conventional linear closure ${ }^{6}$. Another study reported $\mathrm{BMI} \geq 30(\mathrm{OR}=1.4)$, ASA III-IV $(\mathrm{OR}=1.7)$, and operating time $\geq 100 \mathrm{~min}(\mathrm{OR}=3.3)$ as factors associated with incisional infection ${ }^{1}$. We identified in the univariate analysis of our study several factors related with increased SSI included linear wound closure. However, only patients who needed conversion to midline laparotomy were associated with increased likelihood of SSI $(\mathrm{OR}=6.5)$. The rate of conversion to midline incision (9.6\%) seems to be high in our study; the main reason for conversion was dense adhesion needing dissection for adequate tension-free anastomosis.

Mansfield et al. ${ }^{20}$ reported a $7.3 \%$ reoperation rate after ileostomy closure. Schneider et al. ${ }^{23}$ reported an 
immediate reoperation rate after ileostomy reversal of $5.3 \%$, identifying higher $\mathrm{BMI}$ and anemia as the risk factors (BMI: $p=0.038$; OR 0.73 [95\% Cl 0.55-0.98]; anemia: $p=0.001$; OR 25.50 [95\% Cl 3.87-168.21]). Our reoperation rate was $7.3 \%$, and previous use of immunomodulator and performing ileostomy closure through midline incision were found to be independent risk factors.

Medical complications should also be considered after closing a loop ileostomy. In a study including 5401 patients, the frequency of complications was pneumonia $(0.9 \%)$, cardiac $(0.4 \%)$, renal failure $(0.6 \%)$, neurological $(0.1 \%)$, venous thromboembolism $(0.6 \%)$, and urinary tract infections $(2 \%)^{1}$. In one study ${ }^{13}$, age $>80$ years was an independent risk factor for developing urinary retention $(O R=5.6[1.8-17.4] ; p=0.001)$. We found a $9.5 \%$ rate of medical complications, including urinary tract infection (1.5\%), pneumonia (1.5\%), and delirium (1.5\%). Age $\geq 65$ years (OR $=3.5$ ) was an independent risk factor associated with medical complications.

One of the most common late complications of stoma reversal is the development of an incisional hernia, with overall incisional hernia rate of $7.4 \%$, ranging from $0 \%$ to $48 \% \%^{5,24,25}$. Recognized risk factors associated with incisional hernia are age, medical comorbidities (especially, obesity and hypertension), and smoking, as well as the type of suture used to close and $\mathrm{SSI}^{24}$. We found, in our study, an incisional hernia rate of $11 \%$ that is concordant with the incidence reported in previous studies, and two independent risk factors were found to be associated with an increased rate of herniation: time to ileostomy closure $<3$ months $(O R=6.4)$ and presence of parastomal hernia (OR = 13.2). Incisional hernia can have an adverse effect on a patients' quality of life and increases health-care costs $^{5}$. In one study, hernias are detected in a median of 6 months after stoma closure ${ }^{26}$. Around one-third to one-half of hernias detected clinically required repair ${ }^{5}$. Prophylactic mesh placement has been advocated for preventing parastomal hernias; however, it has not been widely used in stoma closure reinforcement due to the risk of contamination ${ }^{5}$. In a recent publication, Warren et al. ${ }^{25}$ advocated the retromuscular placement of permanent synthetic mesh at the time of stoma reversal as an effective mean to prevent the development of incisional hernia.

The limitations of our study are largely attributable to the sample size and the retrospective design. The single institution nature of our investigation is prone to selection bias. The sample size could represent a risk of bias for a multivariate analysis, which could led to the underestimation of the independent variables. However, we consider that this study provides evidence of different risk factors associated with complications that should be considered at the time of loop ileostomy construction and closure either if the first procedure was for a benign or malignant colorectal disease. These data can be utilized by colorectal and general surgeons for surgical decision-making and counseling patients about the risks and complications of having a loop ileostomy.

\section{Conclusions}

Loop ileostomy closure is associated with an important risk of complications either if the first procedure was for a benign or malignant colorectal disease. As loop ileostomy is still recommended for protection of high-risk colorectal anastomosis, several patient-related factors as well as surgical technique elements should be considered at the time of ileostomy closure to reduce post-operative morbidity.

\section{Conflicts of interest}

The authors declare that they have no conflicts of interest.

\section{Funding}

There were no grants/funds used for this research project.

\section{Ethical disclosures}

Protection of human and animal subjects. The authors declare that no experiments were performed on humans or animals for this study.

Confidentiality of data. The authors declare that they have followed the protocols of their work center on the publication of patient data.

Right to privacy and informed consent. The authors declare that no patient data appear in this article.

\section{References}

1. Sharma A, Deeb AP, Rickles AS, et al. Closure of defunctioning loop ileostomy is associated with considerable morbidity. Colorectal Dis. 2013;15:458-62

2. Alberts JC, Parvaiz A, Moran BJ. Predicting risk and diminishing the consequences of anastomotic dehiscence following rectal resection. Colorectal Dis. 2003;5:478-82. 
3. Lightner AL, Pemberton $\mathrm{JH}$. The role of temporary fecal diversion. Clin Colon Rectal Surg. 2017;30:178-83.

4. Gessler B, Haglind E, Angenete E. Loop ileostomies in colorectal cance patients-morbidity and risk factors for nonreversal. J Surg Res. 2012:178:708-14

5. Bhangu A, Nepogodiev D, Futaba K, West Midlands Research Collaborative. Systematic review and meta-analysis of the incidence of incisional hernia at the site of stoma closure. World J Surg. 2012;36:973-83.

6. Hsieh MC, Kuo LT, Chi CC, Huang WS, Chin CC. Pursestring closure versus conventional primary closure following stoma reversal to reduce surgical site infection rate: a meta-analysis of randomized controlled trials. Dis Colon Rectum. 2015;58:808-15.

7. Mennigen R, Sewald W, Senninger N, Rijcken E. Morbidity of loop ileostomy closure after restorative proctocolectomy for ulcerative colitis and familial adenomatous polyposis: a systematic review. J Gastrointest Surg. 2014;18:2192-200

8. Charlson M, Szatrowski TP, Peterson J, Gold J. Validation of a combined comorbidity index. J Clin Epidemiol. 1994;47:1245-51.

9. Dindo D, Demartines N, Clavien PA. Classification of surgical complications: a new proposal with evaluation in a cohort of 6336 patients and results of a survey. Ann Surg. 2004;240:205-13.

10. Mangram AJ, Horan TC, Pearson ML, Silver LC, Jarvis WR. Guideline for prevention of surgical site infection, 1999. Hospital infection contro practices advisory committee. Infect Control Hosp Epidemiol. 1999;20:250-78.

11. Benson $A B$, Venook AP, Al-Hawary MM, et al. NCCN guidelines insights: colon cancer, version 2.2018. J Natl Compr Canc Netw. 2018;16:359-69.

12. Hanna $M H$, Vinci A, Pigazzi A. Diverting ileostomy in colorectal surgery: when is it necessary? Langenbecks Arch Surg. 2015;400:145-52.

13. Man VC, Choi HK, Law WL, Foo DC. Morbidities after closure of ileostomy: analysis of risk factors. Int J Colorectal Dis. 2016;31:51-7.

14. Mirnezami A, Mirnezami R, Chandrakumaran K, et al. Increased local recurrence and reduced survival from colorectal cancer following anastomotic leak: systematic review and meta-analysis. Ann Surg 2011;253:890-9.
15. Konishi T, Watanabe T, Kishimoto J, Nagawa H. Risk factors for anastomotic leakage after surgery for colorectal cancer: results of prospective surveillance. J Am Coll Surg. 2006;202:439-44.

16. Paun BC, Cassie S, MacLean AR, Dixon E, Buie WD. Postoperative complications following surgery for rectal cancer. Ann Surg. 2010; 251:807-18.

17. Rullier E, Laurent C, Garrelon JL, et al. Risk factors for anastomotic leakage after resection of rectal cancer. Br J Surg. 1998;85:355-8.

8. Gu WL, Wu SW. Meta-analysis of defunctioning stoma in low anterio resection with total mesorectal excision for rectal cancer: evidence based on thirteen studies. World J Surg Oncol. 2015;13:9.

19. D'Haeninck A, Wolthuis AM, Penninckx F, D'Hondt M, D'Hoore A. Morbidity after closure of a defunctioning loop ileostomy. Acta Chir Belg. 2011;111:136-41.

20. Mansfield SD, Jensen C, Phair AS, Kelly OT, Kelly SB. Complications of loop ileostomy closure: a retrospective cohort analysis of 123 patients. World J Surg. 2008;32:2101-6.

21. Madani R, Day N, Kumar L, Tilney HS, Gudgeon AM. Hand-sewn versus stapled closure of loop ileostomy: a meta-analysis. Dig Surg. 2018. DOI: 10.1159/000487310 (Article in press).

22. Saha AK, Tapping CR, Foley GT, et al. Morbidity and mortality after closure of loop ileostomy. Colorectal Dis. 2009;11:866-71.

23. Schneider V, Lee LD, Stroux A, et al. Risk factors for reoperation after ileostomy reversal-results from a prospective cohort study. Int J Surg. 2016;36:233-9.

24. Brook AJ, Mansfield SD, Daniels IR, Smart NJ. Incisional hernia following closure of loop ileostomy: the main predictor is the patient, not the surgeon. Surgeon. 2018;16:20-6.

25. Warren JA, Beffa LR, Carbonell AM, et al. Prophylactic placement of permanent synthetic mesh at the time of ostomy closure prevents formation of incisional hernias. Surgery. 2018;163:839-46.

26. Guzmán-Valdivia G. Incisional hernia at the site of a stoma. Hernia. 2008;12:471-4. 